\title{
BMJ open The frequency of EGFR and KRAS mutations in non-small cell lung cancer (NSCLC): routine screening data for central Europe from a cohort study
}

\author{
Christian Boch, ${ }^{1}$ Jens Kollmeier, ${ }^{1}$ Andreas Roth, ${ }^{2}$ Susann Stephan-Falkenau, ${ }^{2}$ \\ Daniel Misch, ${ }^{1}$ Wolfram Grüning, ${ }^{3}$ Torsten Thomas Bauer, ${ }^{1}$ Thomas Mairinger ${ }^{2}$
}

To cite: Boch C, Kollmeier J, Roth $\mathrm{A}$, et al. The frequency of EGFR and KRAS mutations in non-small cell lung cancer (NSCLC): routine screening data for central Europe from a cohort study. BMJ Open 2013:3:e002560.

doi:10.1136/bmjopen-2013002560

- Prepublication history for this paper are available online. To view these files please visit the journal online (http://dx.doi.org/10.1136/ bmjopen-2013-002560).

Received 10 January 2013 Revised 17 February 2013 Accepted 22 February 2013

This final article is available for use under the terms of the Creative Commons Attribution Non-Commercial 2.0 Licence; see http://bmjopen.bmj.com

${ }^{1}$ Klinik für Pneumologie, Lungenklinik Heckeshorn, HELIOS Klinikum Emil von Behring, Berlin, Berlin, Germany

${ }^{2}$ HELIOS Klinikum Emil von Behring, Institut für Gewebediagnostik, Berlin, Berlin, Germany

${ }^{3}$ Klinik für Pneumologie, HELIOS Klinikum Schwerin, Berlin, Berlin, Germany

Correspondence to Professor Torsten Thomas Bauer; torsten.bauer@helioskliniken.de

\section{ABSTRACT}

Objectives: Owing to novel therapy strategies in epidermal growth factor receptor (EGFR)-mutated patients, molecular analysis of the EGFR and KRAS genome has become crucial for routine diagnostics. Till date these data have been derived mostly from clinical trials, and thus collected in pre-selected populations. We therefore screened 'allcomers' with a newly diagnosed non-small cell lung carcinoma (NSCLC) for the frequencies of these mutations.

Design: A cohort study.

Setting: Lung cancer centre in a tertiary care hospital. Participants: Within 15 months, a total of 552 cases with NSCLC were eligible for analysis.

Primary and secondary outcome measures: Frequency of scrutinising exons 18, 19 and 21 for the presence of activating EGFR mutation and secondary codon 12 and 13 for activating KRAS mutations.

Results: Of the 552 patients, 27 (4.9\%) showed a mutation of EGFR. 19 of these patients $(70 \%)$ had deletion E746-A750 in codon 19 or deletion L858R in codon 21. Adenocarcinoma (ACA) was the most frequent histology among patients with EGFR mutations (ACA, 22/254 (8.7\%) vs non-ACA, $5 / 298(1.7 \%) ; p<0.001)$. Regarding only ACA, the percentage of EGFR mutations was higher in women $(16 / 116(14 \%)$ women vs $6 / 138(4.3 \%)$ men; $p=0.008$ ). Tumours with an activating EGFR mutation were more likely to be from non-smokers $(18 / 27 ; 67 \%)$ rather than smoker $(9 / 27 ; 33 \%)$.

KRAS mutation was present in $85(15 \%)$ of all cases. In 73 patients (86\%), the mutation was found in exon 12 and in 12 cases (14\%) in exon 13. Similarly, ACA had a higher frequency of KRAS mutations than non-ACA (67/254 (26\%) vs 18/298 $(6.0 \%) ; p<0.001)$.

Conclusions: We found a lower frequency for EGFR and KRAS mutations in an unselected Caucasian patient cohort as previously published. Taking our results into account, clinical trials may overestimate the mutation frequency for EGFR and KRAS in NSCLC due to important selection biases.

\section{ARTICLE SUMMARY}

Article focus

- Frequency of clinically important mutations in non-small cell lung carcinoma (NSCLC).

- Epidermal growth factor receptor (EGFR) and KRAS.

- Personalised medicine.

Key messages

- The frequency of an activating EGFR mutation can not be expected in more than $4.9 \%$ of the 'allcomers' with the diagnosis of NSCLC.

Strengths and limitations of this study

- Unselected and large cohort of lung cancer centre.

- Analyses of three codons for EGFR.

- Single centre study.

\section{INTRODUCTION}

Lung cancer is still one of the most common cancers in Germany and worldwide, and the most common cause of death due to malignant tumours. ${ }^{1}$ Despite intensive research, the prognosis of patients with non-small cell lung carcinoma (NSCLC) continues to be restricted, which applies especially to patients with distant metastases. However, in patients with NSCLC and an activating mutation of the epidermal growth factor receptor (EGFR), therapy has undergone a significant change. The application of first-generation tyrosine-kinase inhibitors (TKI) Gefitinib an Erlotinib was able to achieve not only a better tolerability of the therapy and an improvement in progressionfree survival in observational studies, ${ }^{2-4}$ but also compared with platinum-based chemotherapy. ${ }^{5}$ However, the effect on overall survival (OS) is still controversial; one study could not detect a benefit, ${ }^{6}$ whereas Zhou and co-workers reported a significant increase in OS. ${ }^{7}$ 
In the last few years, intensive research has been conducted to analyse the effect of TKIs in respect of the complex relationship of biomarkers which are associated with the EGFR pathway like v-Ki-ras2 Kirsten rat sarcoma viral oncogene homologue (KRAS). In addition to the superiority in EGFR-mutated patients, TKIs seem to have an effect on the wild-type NSCLC as well, while some studies have disputed this for KRAS mutated patients. ${ }^{2} 8$ However, the significance of KRAS mutations for the diagnosis or treatment of NSCLC remains under debate. Since double mutations (EGFR and KRAS) have been described in only very few cases, KRAS may help exclude EGFR mutation. ${ }^{9} 10$

Therefore, the question arises as to how many patients with NSCLC can benefit from the new treatment option and whether screening should be implemented in the routine diagnostics. In addition, numerous studies came from Southeast Asia, where patients with NSCLC have higher EGFR mutation rates. ${ }^{11}{ }^{12}$ Since the data available today are hampered either by biases owing to study exclusion criteria or different geographical peculiarities, we established routine screening for consecutive patients to receive reliable data for the routine setting in Central Europe.

\section{PATIENTS AND METHODS}

\section{Study population}

From October 2009 until December 2010 we have screened prospectively all newly diagnosed patients with NSCLC ( $\mathrm{n}=753)$ for the ability to be analysed for EGFR and KRAS mutations. All subsequent biopsies have been obtained by surgery, routine bronchoscopy or CT-guided biopsy. Staging of the tumour war performed according to the Union for International Cancer Control (UICC, Seventh Edition). ${ }^{13}$ The complete staging was available for $735 / 753$ patients $(97.6 \%)$. The histological diagnosis was performed according to the WHO criteria. ${ }^{14} \mathrm{~A}$ total of 552/753 (75\%) cases with NSCLC were eligible for analysis. In 183 patients (25\%) none of the two analyses could be performed. In the majority of cases, this was explained by an insufficient amount of tissue sample. In addition, some patients refused their consent to carry out the mutation analysis. Informed consent was obtained from all patients prior to biopsy.

\section{DNA extraction}

After preparation of a $20 \mu \mathrm{m}$ thick section from the formalin-fixed and paraffin-embedded tissue sample, the area of tumour cells was marked by comparison with the corresponding HE-stained slide. This area with a size of $2-10 \mathrm{~mm}^{2}$ was scraped with a pipette tip and transferred into $180 \mu \mathrm{l}$ AL-buffer. The AL-buffer is included in the QIAamp DNA Mini Kit (Qiagen, Hilden, Germany). Later the DNA was extracted and dissolved in $150 \mu \mathrm{l} \mathrm{AE-buffer} \mathrm{according} \mathrm{to} \mathrm{the} \mathrm{manufacturer's}$ instructions using the QIAamp DNA Mini Kit.

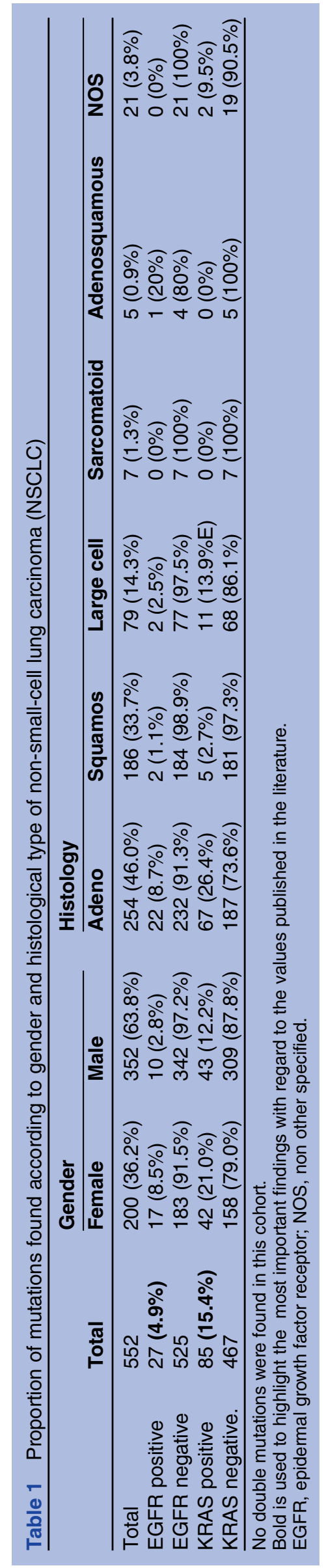




\section{Determination of EGFR mutations}

For analysing the EGFR mutation status, the extracted DNA was subjected to conventional PCR for amplification of relevant portions of exons 18, 19 and 21 of the EGFR gene. Further, the PCR products were analysed for the presence of a mutation by using the Pyrosequencing PyroMark Instrument (Qiagen, Hilden, Germany). The primers used for the PCR are listed in table 1. The method of pyrosequencing has been performed according to the reagent manufacturer (Qiagen, Hilden, Germany) and the following primer names and sequences $\left(3^{\prime}-5^{\prime}\right)$ have been used for exons 18, 19 and 21:

\section{- 18_F (forward) CAGCATGGTGAGGGCTGAG \\ - 18_R (reverse) GCCTGTGCCAGGGACCTTAC \\ - 19_F (forward) GTGGCACCATCTCACAATTGC \\ - 19_R (reverse) CACACAGCAAAGCAGAAACTCAC \\ - 21_F (forward) CCTCACAGCAGGGTCTTCTCTG \\ - 21_R (reverse) CTGGCTGACCTAAAGCCACC}

Samples with a mutation in the pyrogram were additionally examined by dideoxy sequencing according to Sanger et $a l^{15}$ for confirmation (figure 1).

\section{Determination of KRAS mutations}

The DNA was analysed according to manufacturer's instructions using the K-RAS codon 12/13 kit from Molbiol TIB (Berlin, Germany) and the LightCycler 2.0 instrument (Roche Diagnostics, Mannheim, Germany).

This is a real-time PCR with FRET probes, which facilitate the differentiation of mutant and wild-type DNA in codons 12 and 13 on exon 1 of KRAS gene after successful amplification and analyses of the melting curve of the PCR products.

\section{Statistical analyses}

Quantitative variables were summarised as means \pm SD. For comparisons Student $\mathrm{t}$ test was used and in cases with more than two groups, a one-way analysis of variance (ANOVA) with Bonferroni correction was calculated. Categorical variables are reported as frequencies and for comparisons of categorical variables between groups $\chi^{2}$ tests were used. The level of significance for all tests was set to $5 \%$ and all tests were two-sided. Analyses were carried out with the Statistical Package for Social Sciences (SPSS, V.13.0) on a Windows operating system.

\section{RESULTS}

\section{Patients}

The mean age of patients with wild-type EGFR $(n=440$; $66.7 \pm 9.5$ years) was comparable with the mean age of patients with EGFR mutation ( $\mathrm{n}=27 ; 70.3 \pm 11.4$ years) and that of patients with a KRAS mutation in the overall comparison $(\mathrm{n}=85 ; 65.3 \pm 9.8$ years; $\mathrm{p}=0.059)$. Table 1 summarises frequencies according to mutation, gender and histology.

A breakdown of patients according to the genealogy was not performed because all patients with a migration background except two came from other European countries. Patients from Asia were not diagnosed or treated in our clinic during the corresponding period.

\section{EGFR mutation}

Overall $27(4.9 \%)$ of all 552 patients showed an activating EGFR mutation (3/27 mutations in exon $18(11 \%)$, $15 / 27$ mutations in exon $19(56 \%)$ and 8/27 mutations $(30 \%)$ in exon $21 ; 1 / 27$ tumour $(3 \%)$ has shown a double mutation (exons 19 and 21)). The most common mutation in exon 19 was delE746-A750 with $10 / 15(67 \%)$ cases. In exon 21, all detected eight mutations were located on L848R (100\%).

Considering the frequency of mutations according to histology, adenocarcinomas (ACAs) show mutations more often than any other histology (22/254 (8.7\%) vs $5 / 298(1.7 \%) ; \mathrm{p}<0.001)$.

When all histologies were analysed together, women also showed a significantly higher frequency of EGFR mutation compared with men $(17 / 200(8.5 \%)$ vs $10 /$ $352(2.8 \%) ; \mathrm{p}=0.003$; table 2 upper and lower panels).

However, when the subgroup of ACAs was analysed among women, the higher mutation rate compared with histologies remained (16/116 (14\%) vs $1 / 84(1.2 \%)$, $\mathrm{p}=0.002$; table 2 upper panel). In contrast, among men with NSCLC no significant difference in the rate of mutation could be detected with regard to histology (ACA, 6/138 (4.3\%) vs non-ACA, 4/214 (1.9\%); $\mathrm{p}=0.172$; table 2 lower panel).

Smoking status was closely associated with EGFR mutation rate. Among patients with EGFR mutations ( $n=27)$ the rate of never-smokers was twice as high compared with smokers (18/27 (67\%) vs 9/27 (33\%); table 3).

\section{KRAS mutation}

A mutation in the KRAS gene was seen in a total of 85/ $552(15 \%)$ of all analysed tumours. While 73/85 (86\%) of these mutations were found in exon 12, in exon 13
Figure 1 Representation of a mutation in exon 19 (del E746-A750) by using Sanger dideoxy ${ }^{15}$.

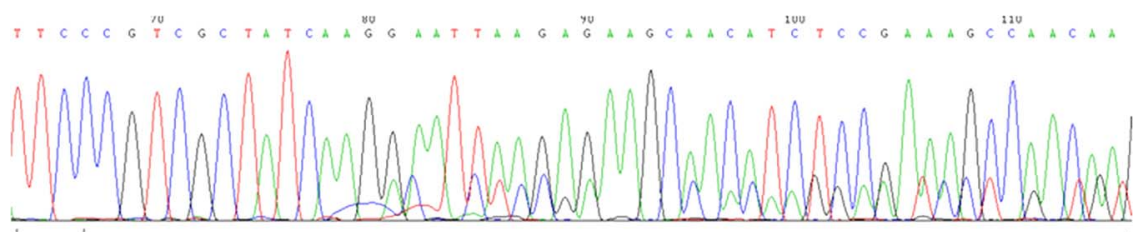


Table 2 Proportion of EGFR and KRAS mutations found according to women (upper table) and men (lower table)

\begin{tabular}{|c|c|c|c|c|c|}
\hline \multirow[b]{2}{*}{ Total number of women } & \multirow{2}{*}{$\begin{array}{l}\text { Total } \\
200\end{array}$} & \multicolumn{2}{|c|}{ EGFR positive (\%) } & \multicolumn{2}{|c|}{ KRAS positive (\%) } \\
\hline & & 17 & 8.5 & 42 & 21.0 \\
\hline Adenocarcinoma & 116 & 16 & 13.8 & 31 & 26.7 \\
\hline Squamous cell carcinoma & 47 & 0 & 0.0 & 2 & 4.3 \\
\hline Large cell carcinoma & 28 & 1 & 3.6 & 7 & 25.0 \\
\hline Sarcomatoid carcinoma & 0 & 0 & 0.0 & 0 & 0.0 \\
\hline Adenosquamous carcinoma & 0 & 0 & 0.0 & 0 & 0.0 \\
\hline NOS (non other specified) & 9 & 0 & 0.0 & 2 & 22.2 \\
\hline Total number of men & 352 & 10 & 2.8 & 43 & 12.2 \\
\hline Adenocarcinoma & 138 & 6 & 4.3 & 36 & 26.1 \\
\hline Squamous cell carcinoma & 139 & 2 & 1.4 & 3 & 2.2 \\
\hline Large cell carcinoma & 51 & 1 & 2.0 & 4 & 7.8 \\
\hline Sarcomatoides Karzinom & 7 & 0 & 0.0 & 0 & 0.0 \\
\hline Adenosquamous carcinoma & 5 & 1 & 20.0 & 0 & 0.0 \\
\hline NOS (non other specified) & 12 & 0 & 0.0 & 0 & 0.0 \\
\hline
\end{tabular}

only 12/85 (14\%) mutations were localised. With 29/85 (34\%) cases the 12 Cys mutation was the most common. Similar to the EGFR the subgroups of ACAs had significantly $(\mathrm{p}<0.001)$ higher rate of KRAS mutations compared with other histologies $(67 / 254$ (26\%) vs 18/298 $(6 \%)$; table 1). Within the subgroup of ACAs there was an even gender distribution regarding the frequency of KRAS mutations (women, 31/116 (27\%) vs men, 36/138 (26\%); $\mathrm{p}=0.909$; table 2 upper and lower panels).

However, regardless of histology the rate of KRAS mutations was significantly $(\mathrm{p}=0.007)$ more common in women than in men $(42 / 200(21 \%)$ vs $43 / 352(12 \%))$. Besides that, the KRAS mutation in large cell carcinomas was significantly $(\mathrm{p}<0.001)$ more frequent compared with squamous cell carcinomas $(11 / 80$ (14\%) vs 5/186 $(2.7 \%)$; table 1$)$. No patient had a double mutation (KRAS and EGFR).

Unlike in patients with an activating EGFR mutation the proportion of smokers is higher in patients with a KRAS mutation $(75 / 85(88 \%)$ vs $10 / 85$ (22\%); table 3$)$.

\section{Intention-to-treat analyses}

Table 4 concludes the distribution according to histology and gender for all patients not included in this analysis $(n=180)$. The percentage of tumours that had to be left unclassified (NOS; non other specified) was substantially higher $(38 / 180,21 \%)$ compared with the patient group included $(21 / 552 ; 3.8 \% ; \mathrm{p}<0.001)$. This was most likely owing to an insufficient probe size, which hampered both plain histology and mutation analysis. The percentage of patients with ACAs excluded was slightly lower (39\% vs $46 \%)$, however, this difference was not statistically significant $(\mathrm{p}=0.112$; tables 1 and 4$)$.

When we analysed the distribution according to the clinical stage, we found a bias with regard to higher clinical stages in the non-tested group (table 5). However, when stages IIIB and IV were compared with the potentially operable stages (IA-IIIA) we found this difference not to be statistically significant (not tested: 102/180; $56.7 \%$ vs tested: $277 / 552 ; 50.2 \%, \mathrm{p}=0.077)$.

\section{DISCUSSION}

The present study delivers sound data on the frequency of EGFR and KRAS mutations in a large and unselected cohort of patients with newly diagnosed non-small cell lung cancer (NSCLC) over a well-defined study period. The EGFR mutation rate reported here $(4.9 \%)$ differs largely from frequencies reported in previous studies $(8-60 \%))^{2} 516$ This suggests that previous study designs had import selection biases concerning this mutation analyses. In the most extensive study on the prevalence of activating EGFR mutations in NSCLC at 126 Spanish hospitals during the years 2005-2008, a total of 2105

Table 3 Smoker status and gender distribution of all 112 patients with either EGFR or KRAS

\begin{tabular}{|c|c|c|c|c|}
\hline \multirow[b]{2}{*}{ Total number of mutations } & \multicolumn{2}{|c|}{ Smokers } & \multicolumn{2}{|c|}{ Never-smokers } \\
\hline & 84 & $75.0 \%$ & 28 & $25.0 \%$ \\
\hline EGFR positive & 9 & $33.3 \%$ & 18 & $66.7 \%$ \\
\hline EGFR positive female & 5 & $29.4 \%$ & 12 & $70.6 \%$ \\
\hline EGFR positive male & 4 & $40.0 \%$ & 6 & $60.0 \%$ \\
\hline KRAS positive & 75 & $88.2 \%$ & 10 & $11.8 \%$ \\
\hline KRAS positive female & 38 & $90.5 \%$ & 4 & $9.5 \%$ \\
\hline KRAS positive male & 37 & $86.0 \%$ & 6 & $14.0 \%$ \\
\hline
\end{tabular}

Bold is used to highlight the most important findings with regard to the values published in the literature. 
Table 4 Histology of the patients not included in the study, because of insufficient probe size or denial of informed consent

\begin{tabular}{lrrrr}
\hline & \multicolumn{3}{c}{ Per } \\
& Total & cent & Men & Women \\
\hline Total & 180 & & 111 & 69 \\
Adenocarcinoma & 70 & 38.9 & 38 & 32 \\
Squamous cell carcinoma & 47 & 26.1 & 34 & 13 \\
Large cell carcinoma & 17 & 9.4 & 10 & 7 \\
Sarcomatoides Karzinom & 8 & 4.4 & 7 & 1 \\
Adenosquamous & 0 & 0.0 & 0 & 0 \\
carcinoma & & & & \\
NOS (non other specified) & 38 & 21.1 & 22 & 16 \\
\hline
\end{tabular}

patients were analysed and a mutation rate of $16.6 \%$ was reported. ${ }^{17}$ One possible explanation for the higher mutation rate in this study lies in the unrepresentative distribution of the histological subgroups. While the expected proportion of ACAs in routine studies is between $32 \%$ and $54 \%$ depending on the literature, this study reported up to $78 \%$ ACAs. Since ACAs were also in our study more likely to exhibit an activating EGFR mutation, the frequency was overestimated in the Spanish study. ${ }^{17}$ The disproportion of ACAs is also found in other studies reporting EGFR mutation frequencies between $9 \%$ and $31 \% .^{218} 19$ In our analysis, however, the proportion of ACAs was well within the expected range $(46 \%)$ with no selection bias induced by patient selection.

Another factor with great influence on the EGFR mutation rate is the geographical origin of the analysed patients. For quite some years it has been known that East Asian patients with NSCLC have a higher incidence of EGFR mutations while they show lower incidence of KRAS mutations compared with other ethnic groups at the same time. ${ }^{12} 2021$ Studies with a high proportion of patients from Far Eastern countries or studies which have recruited their patients entirely in the East Asian region have consequently shown a corresponding effect on the frequency of the detected EGFR and KRAS mutations. ${ }^{6223}$ None of our patients with a migration background came from an East Asian country and thus our figures are not prone to this bias.
A study from Basel seems to be the most comparable in terms of patient's cohort. Pathologists examined 307 NSCLC during a 4.5-year period for EGFR mutation and showed a mutation rate of $8.1 \%$. However it remains unclear in this study, which preliminary selection bias may have been introduced by the clinicians, because mutation analyses were not requested for all samples. Moreover, it has not been reported how many patients with NSCLC were newly diagnosed in the participating hospitals. $^{16}$

As demonstrated in our study, patients with NSCLC and EGFR mutation are non-smokers in the majority of cases, which has been confirmed by numerous studies. ${ }^{5} 2425$ In Europe and in Germany as well it is estimated that $85 \%$ of lung cancers-related deaths are associated with cigarette smoking. ${ }^{26}$ Such a high proportion of smokers among patients with lung cancer make a high EGFR mutation rate questionable. However, the question arises why this mutation occurs much more frequently in the Asian populations.

Independent of the rate of mutation in total, the frequency of EGFR mutations in exons 19 and 21 of our samples is comparable with those of numerous studies. $^{22}$ 27-29 In addition, the occurrence of the most common mutation in our evaluation delE746-A750 in exon $19(10 / 27(37 \%))$ corresponds to the literature. ${ }^{30}$ Another interesting aspect is that the restriction on the search for the two most common mutations in exons 19 (delE746-A750) and 21 (L858R), as discussed in the literature, would have detected only $18 / 27(69.2 \%)$ of all EGFR mutations in our study.

Regarding the KRAS mutations our analysis confirms the known fact that KRAS mutations occur in smokers with NSCLC significantly more often than in nonsmokers. ${ }^{31} 32$ This is similar to the distribution of mutations of KRAS at codon $12(72 / 85(85 \%))$ and $13(13 / 85$ $(15.3 \%))$, which appears to be comparable with the frequency of the other studies. ${ }^{53}$ Therefore, our results regarding the frequency of KRAS mutation are in keeping with the published reports. The missing gender bias and the higher frequency among smokers repeated in our sample support the validity of our data. Till date, the clinical applicability of the KRAS mutation is limited, because

Table 5 Lung cancer stages of the investigated cohort and the excluded patients (according to UICC 7 th edition) ${ }^{13}$

\begin{tabular}{|c|c|c|c|c|c|c|}
\hline & $\begin{array}{l}\text { Intention to treat } \\
\text { population }(n=732)\end{array}$ & Per cent & $\begin{array}{l}\text { Study Population } \\
(n=522)\end{array}$ & Per cent & $\begin{array}{l}\text { Patients excluded from } \\
\text { analyses }(n=180)\end{array}$ & Per cent \\
\hline \multicolumn{7}{|l|}{ Clinical Stage (n, \%) } \\
\hline Staging not completed & 13 & 1.8 & 9 & 1.6 & 4 & 2.2 \\
\hline IA & 76 & 10.4 & 56 & 10.1 & 20 & 11.1 \\
\hline IB & 50 & 6.8 & 36 & 6.5 & 14 & 7.8 \\
\hline IIA & 44 & 6.0 & 35 & 6.3 & 9 & 5.0 \\
\hline IIB & 54 & 7.4 & 44 & 8.0 & 10 & 5.6 \\
\hline IIIA & 116 & 15.8 & 95 & 17.2 & 21 & 11.7 \\
\hline IIIB & 82 & 11.2 & 70 & 12.7 & 12 & 6.7 \\
\hline IV & 297 & 40.6 & 207 & 37.5 & 90 & 50.0 \\
\hline
\end{tabular}


there are no clinical associations strong enough for inclusion in clinical or therapeutic algorithms. However, KRAS mutation exclude or are only very rarely associated with other mutations (eg, EGFR). KRAS may therefore help to build up a testing algorithm in order to get a reasonable genetic array for this type of cancer.

In summary, for the first time we offer unbiased mutation rates for EGFR and KRAS in an unselected cohort of Caucasian patients. Previously described mutation rates for EGFR need to be corrected to approximately $5 \%$, thus lowering the proportion of patients with NSCLC entitled to personalised medicine in Central Europe. However, in keeping with the literature nonsmoking women with ACA do have the highest frequency of tumours with an activating EGFR mutation.

Contributors $\mathrm{CB}, \mathrm{JK}$ and TM worked on the idea of the study and were responsible for the design and the setup. AR, SSF, DM and WG organised patient specimens and analyses and thus produced the data and were responsible for its analysis in tables and graphs. CB, JK, DM and TTB performed data analyses and wrote the initial manuscript. All authors contributed to the final manuscript and the interpretation of the data. All authors read and approved the final manuscript.

Funding Oskar-Helene Heim Foundation, Berlin, Germany. Competing interests None.

Ethics approval Routine informed consent was obtained for the diagnostic procedure.

Provenance and peer review Not commissioned; externally peer reviewed.

Data sharing statement No additional data are available.

\section{REFERENCES}

1. Jemal A, Siegel R, Xu J, et al. Cancer statistics, 2010. CA Cancer J Clin 2010;60:277-300.

2. Van Zandwijk N, Mathy A, Boerrigter L, et al. EGFR and KRAS mutations as criteria for treatment with tyrosine kinase inhibitors: retro- and prospective observations in non-small-cell lung cancer. Ann Oncol 2007;18:99-103.

3. Schneider CP, Heigener D, Schott-von-Romer K, et al. Epidermal growth factor receptor-related tumor markers and clinical outcomes with erlotinib in non-small cell lung cancer: an analysis of patients from german centers in the TRUST study. $J$ Thorac Oncol 2008;3:1446-53.

4. Heigener DF, Wu YL, Van ZN, et al. Second-line erlotinib in patients with advanced non-small-cell lung cancer: subgroup analyses from the TRUST study. Lung Cancer 2011;74:274-9.

5. Mok TS, Wu YL, Thongprasert S, et al. Gefitinib or carboplatin-paclitaxel in pulmonary adenocarcinoma. $N$ Engl J Med 2009;361:947-57.

6. Douillard JY, Shepherd FA, Hirsh V, et al. Molecular predictors of outcome with gefitinib and docetaxel in previously treated non-small-cell lung cancer: data from the randomized phase III INTEREST trial. J Clin Oncol 2010;28:744-52.

7. Zhou C, Wu YL, Chen G, et al. Erlotinib versus chemotherapy as first-line treatment for patients with advanced EGFR mutation-positive non-small-cell lung cancer (OPTIMAL, CTONG-0802): a multicentre, open-label, randomised, phase 3 study. Lancet Oncol 2011;12:735-42.

8. Massarelli $\mathrm{E}$, Varella-Garcia $\mathrm{M}$, Tang $\mathrm{X}$, et al. KRAS mutation is an important predictor of resistance to therapy with epidermal growth factor receptor tyrosine kinase inhibitors in non-small-cell lung cancer. Clin Cancer Res 2007;13:2890-6.

9. Boldrini L, Ali G, Gisfredi S, et al. Epidermal growth factor receptor and K-RAS mutations in 411 lung adenocarcinoma: a population-based prospective study. Oncol Rep 2009;22:683-91.

10. Eberhard DA, Johnson BE, Amler LC, et al. Mutations in the epidermal growth factor receptor and in KRAS are predictive and prognostic indicators in patients with non-small-cell lung cancer treated with chemotherapy alone and in combination with erlotinib. $J$ Clin Oncol 2005;23:5900-9.

11. Wu YL, Zhong WZ, Li LY, et al. Epidermal growth factor receptor mutations and their correlation with gefitinib therapy in patients with non-small cell lung cancer: a meta-analysis based on updated individual patient data from six medical centers in mainland China. J Thorac Oncol 2007;2:430-9.

12. Sriuranpong V, Chantranuwat $\mathrm{C}$, Huapai $\mathrm{N}$, et al. High frequency of mutation of epidermal growth factor receptor in lung adenocarcinoma in Thailand. Cancer Lett 2006;239:292-7.

13. International Association for the Study of Lung Cancer. Staging manual in thoracic oncology. Florida, USA: Editorial Rx Press, 2009.

14. Travis WD, Garg K, Franklin WA, et al. Bronchioloalveolar carcinoma and lung adenocarcinoma: the clinical importance and research relevance of the 2004 World Health Organization pathologic criteria. $J$ Thorac Oncol 2006;1(9 Suppl):S13-19.

15. Sanger $F$, Nicklen $S$, Coulson AR. DNA sequencing with chain-terminating inhibitors. Proc Natl Acad Sci USA 1977;74:5463-7.

16. Tapia C, Savic S, Bihl M, et al. [EGFR mutation analysis in non-small-cell lung cancer: Experience from routine diagnostics]. Pathologe 2009;30:384-92.

17. Rosell R, Moran T, Queralt C, et al. Screening for epidermal growth factor receptor mutations in lung cancer. $N$ Engl J Med 2009;361:958-67.

18. Hirsch FR, Varella-Garcia M, Cappuzzo F, et al. Combination of EGFR gene copy number and protein expression predicts outcome for advanced non-small-cell lung cancer patients treated with gefitinib. Ann Oncol 2007:18:752-60.

19. Khambata-Ford S, Harbison CT, Hart LL, et al. Analysis of potential predictive markers of cetuximab benefit in BMS099, a phase III study of cetuximab and first-line taxane/carboplatin in advanced non-small-cell lung cancer. J Clin Oncol 2010;28:918-27.

20. Pirker R, Pereira JR, Szczesna A, et al. Cetuximab plus chemotherapy in patients with advanced non-small-cell lung cancer (FLEX): an open-label randomised phase III trial. Lancet 2009;373:1525-31.

21. Sakuma Y, Matsukuma S, Yoshihara M, et al. Distinctive evaluation of nonmucinous and mucinous subtypes of bronchioloalveolar carcinomas in EGFR and K-ras gene-mutation analyses for Japanese lung adenocarcinomas: confirmation of the correlations with histologic subtypes and gene mutations. Am J Clin Pathol 2007;128:100-8.

22. Brugger W, Triller N, Blasinska-Morawiec M, et al. Prospective molecular marker analyses of EGFR and KRAS from a randomized, placebo-controlled study of erlotinib maintenance therapy in advanced non-small-cell lung cancer. J Clin Oncol 2011;29:4113-20.

23. Li M, Liu L, Liu Z, et al. The status of KRAS mutations in patients with non-small cell lung cancers from mainland China. Oncol Rep 2009;22:1013-20.

24. Scoccianti C, Vesin A, Martel G, et al. Prognostic value of TP53, KRAS and EGFR mutations in nonsmall cell lung cancer: the EUELC cohort. Eur Respir J 2012;40:177-84.

25. Ren JH, He WS, Yan GL, et al. EGFR mutations in non-small-cell lung cancer among smokers and non-smokers: a meta-analysis. Environ Mol Mutagen 2012;53:78-82.

26. Bogdanovica I, Godfrey F, Mcneill A, et al. Smoking prevalence in the European Union: a comparison of national and transnational prevalence survey methods and results. Tob Control 2011;20:e4.

27. Sharma SV, Bell DW, Settleman J, et al. Epidermal growth factor receptor mutations in lung cancer. Nat Rev Cancer 2007;7:169-81.

28. Bonanno L, Schiavon M, Nardo G, et al. Prognostic and predictive implications of EGFR mutations, EGFR copy number and KRAS mutations in advanced stage lung adenocarcinoma. Anticancer Res 2010;30:5121-8.

29. Reinersman JM, Johnson ML, Riely GJ, et al. Frequency of EGFR and KRAS mutations in lung adenocarcinomas in African Americans. J Thorac Oncol 2011;6:28-31.

30. Lynch TJ, Bell DW, Sordella R, et al. Activating mutations in the epidermal growth factor receptor underlying responsiveness of non-small-cell lung cancer to gefitinib. $N$ Engl $J$ Med 2004:350:2129-39.

31. Nelson HH, Christiani DC, Mark EJ, et al. Implications and prognostic value of K-ras mutation for early-stage lung cancer in women. J Natl Cancer Inst 1999;91:2032-8.

32. Ahrendt SA, Decker PA, Alawi EA, et al. Cigarette smoking is strongly associated with mutation of the K-ras gene in patients with primary adenocarcinoma of the lung. Cancer 2001;92:1525-30.

33. Siegfried JM, Gillespie AT, Mera R, et al. Prognostic value of specific KRAS mutations in lung adenocarcinomas. Cancer Epidemiol Biomarkers Prev 1997;6:841-7. 